\title{
REVIEW
}

\section{Pseudomonas aeruginosa wound infections: a critical appraisal of topical antiseptics}

\author{
Haley Augustine MScOT MD', Joshua Gillis MD², Jason Williams MEd MD² \\ 'Class of 2015, Faculty of Medicine, Dalhousie University \\ ${ }^{2}$ Division of Plastic and Reconstructive Surgery, Faculty of Medicine, Dalhousie University
}

\begin{abstract}
Background: Pseudomonas aeruginosa infection is a serious complication of burns and wounds with a high degree of morbidity and mortality. This paper reviews the literature on the use of topical antiseptics for the treatment of $P$. aeruginosa wound infections.

Methods: Embase and PubMed were searched, yielding 119 results that were reviewed. Inclusion criteria were all papers that assessed patients with confirmed $P$. aeruginosa wound infection treated with topical antiseptics and reported the eradication of the pathogen.

Results: Three papers met the inclusion criteria, with a total sample size of 33. All of the studies analyzed acetic acid for the treatment of $P$. aeruginosa wound infection. The pooled data demonstrated that $84.8 \%$ of confirmed $P$. aeruginosa wound infections were effectively treated with acetic acid.

Conclusions: $P$. aeruginosa remains a serious infection complicating burn and wound healing. Treatment commonly includes topical antiseptics; however, there is a paucity of clinical based trials to support this. Three small trials demonstrated some evidence for the use of acetic acid as a topical treatment. More evidence is required to demonstrate the efficacy of topical antiseptics as well as recommend specific types of topical treatment for this pathogen.
\end{abstract}

Tnfection is the main cause of delayed wound healing 1 in primary closure, traumatic wounds, burns, and chronic skin ulcers. ${ }^{1}$ Pseudomonas aeruginosa is an opportunistic infectious pathogen that poses a management challenge while carrying significant morbidity and mortality. ${ }^{1-4}$ Part of the challenge is the fact that excessive antibiotic use can promote the selection and multiplication of resistant isolates. $P$. aeruginosa is a ubiquitous nosocomial infection in burn patients and multi-drug resistant strains are common in burn units, leading to a high mortality rate. It is the most common cause of bacteremia in burn patients, and $14-33 \%$ of burn wounds are colonized with P. aeruginosa within 10 days of admission., ${ }^{1,3-5}$

Risk of infection in burn patients is related to the surface area of the burn (>30\% total body area), preexisting disease, or complications preventing surgical excision of the eschar or closure of the wound. ${ }^{1,6}$ The key to prevention and treatment of burn wound sepsis is rapid and thorough debridement. P. aeruginosa permeates subcutaneous lymphatics, multiplies and invades the surrounding viable adjacent tissue. ${ }^{7}$ The poorly perfused avascular tissue does not benefit from systemic therapies, therefore topical antiseptics agents are routinely used to assist in the eradication of $P$. aeruginosa wound and burn infections. ${ }^{1,8}$ Tolerability must be balanced with the antimicrobial activity. Ideal topical antimicrobials reduce colony-forming units without inhibiting cellular wound healing. ${ }^{1}$

There are a variety of topical antimicrobials or antiseptics used to treat $P$. aeruginosa wound infection. Examples include acetic acid, silver based topical treatments, and mafenide acetate. Agents such as mafenide acetate are reported to be extremely painful to apply, and may result in a metabolic acidosis and hyperventilation secondary to its activity as a carbonic anhydrase inhibitor. ${ }^{8,9}$ However, mafenide acetate is water-soluble and can penetrate the burn eschar and underlying tissues to reduce microbial density. ${ }^{6}$

Silver based therapies have silver ions that react with sulfhydryl groups of bacterial enzymes and deoxyribonucleic acid, to elicit bactericidal and bacteriostatic action. ${ }^{4,9}$ Silver sulfadiazine is a relatively safe, simple and effective agent against a range of gram- negative and positive organisms. However, it is considered less penetrative and has less antibacterial effectiveness than other topical treatments. ${ }^{8}$ Similarly, silver nitrate has not been proven effective in established burn wound infection, is nonpenetrative and can complicate fluid and electrolyte management with leaching of ions into the dressing. However, proponents of silver nitrate claim it has moderate effective antimicrobial properties, is painless, and decreases heat loss..$^{1,8,10}$ 
Acetic acid is another topical antiseptic that has been used to treat chronic wounds, and has shown efficacy in treating gram-negative bacteria, especially $P$. aeruginosa. In vitro studies have demonstrated toxicity to fibroblasts; however, when studied in animal models, acetic acid was well tolerated and effective against $P$. aeruginosa. ${ }^{3,11}$ A concentration of at least $0.5 \%$ is required for clinical antimicrobial efficacy, and the efficacy is suggested to be secondary to a lowering of the $\mathrm{pH}$ to make the wound environment unsuitable for P. aeruginosa growth. ${ }^{3,12}$

In our centre, over a 17-year period from 1996 to 2012 , there were a total of 46 confirmed burns complicated with pseudomonas infection (9.62\%). Of those confirmed infections, the majority (34 of 46 infections [74\%]) were treated with acetic acid (Table $1)$. Due to the significant morbidity of $P$. aeruginosa, this study was performed to evaluate and compare the different topical antimicrobial treatments for wound or burn infections complicated by P. aeruginosa in order to guide future treatment approaches.

\begin{tabular}{lc}
\hline Treatment & Frequency (\%) \\
\hline Acetic Acid & $34(73.91 \%)$ \\
Silver-containing & $6(13.04 \%)$ \\
Other & $4(8.70 \%)$ \\
Unknown & $1(2.17 \%)$ \\
None & $1(2.17 \%)$ \\
Total & $46(100.0 \%)$ \\
\hline
\end{tabular}

Table 1. Summary of topical antimicrobial treatments for Pseudomonas burn wound infections from the Halifax Infirmary burn ward, Halifax, Nova Scotia from 1996 to 2012.

\section{Literature Review}

All published data assessing topical antimicrobials for the treatment of P. aeruginosa wound infections were reviewed with no limits applied for year of publication. Embase and PubMed databases were accessed on June 25, 2014 using the following search criteria, specifying human studies: pseudomonas, wound, mafenide acetate, sulfadizine silver, silver nitrate, silvercel, acetic acid, wound infection, local anti-infective agents, and burns.

This search string yielded 119 results from Embase and 971 results from PubMed, three of which were included in this review (Figure 1). The inclusion criteria were defined as patients with confirmed $P$. aeruginosa wound infection (burn, wound, ulcer, or unspecified), treated with topical antiseptics (acetic acid, silver containing topicals, mafenide, or other), and the study had to report outcomes from treatment, specifically the eradication of $P$. aeruginosa. Studies were excluded if they did not specify the microbial pathogen as $P$. aeruginosa, the design of the study was in vitro or in vivo, if they were non-human studies, topical antimicrobials were used as prophylactic prevention of infection, or the studies did not report sufficient data (details of treatment, patient population, intervention, and outcomes). Case reports were excluded, however were commented on in the discussion.

\section{Results}

Three studies met the inclusion and exclusion criteria, described above, and are summarized in Table 2. In the prospective, randomized trial by Phillips et al. patients with confirmed $P$. aeruginosa wound infection were randomized to either acetic acid or chlorohexidine/ hypochlorite treatment groups. ${ }^{13}$ Ten patients were included in each group and they received a course of seven days of treatment in addition to antibiotics. After seven days of treatment with acetic acid 7/10 patients had no evidence of infection, compared to $1 / 10$ in the chlorohexidine/hypochlorite group (P-value not stated).

In the prospective study by Sloss et al. eight patients with $P$. aeruginosa infected ulcers and eight burn wound infections were treated with topical 1-5\% acetic acid, along with $0.5 \%$ soaks for the burn patients. ${ }^{5}$ All of the patients tolerated the treatment well; however, some complained about stinging on application of the $5 \%$ solution, but this did not interrupt treatment. The authors found that the $5 \%$ concentration of acetic acid was the most effective for eliminating $P$. aeruginosa and that the time to elimination ranged from 2 to 14 days. Of the patients included in this study, 14 of 16 were effectively treated with acetic acid (P-value not stated).

The prospective study by Nagoba et al. included seven patients that had $P$. aeruginosa wound infections not responding to traditional therapy for more than 10 days..$^{12} P$. aeruginosa was isolated from the exudate and in vitro susceptibility of acetic acid was tested. The susceptibility was tested by culturing the bacteria with nutrient broth and 1-5\% acetic acid for varying time points and then plated and incubated. The lowest concentration of acetic acid to inhibit growth was recorded. No antibiotics were given to the patients during the course of topical treatment. They found that the isolated strains of $P$. aeruginosa were resistant to four or more antibacterial agents; however, six of the seven cases were inhibited by $3 \%$ acetic acid in vitro. All patients were thus successfully treated with topical $3-5 \%$ acetic acid within a range of 2-12 applications without any adverse effects (P-value not stated). 


\begin{tabular}{lcccccc}
\hline Study & Design & $\begin{array}{c}\text { Infection } \\
\text { type }\end{array}$ & $\begin{array}{c}\text { Treatment } \\
\text { duration }\end{array}$ & $\begin{array}{c}\text { Sample } \\
\text { size }\end{array}$ & Treatment & Eradication \\
rate
\end{tabular}

Table 2. Description of the studies that met inclusion criteria and summary of results.

Pooled data from these studies had total sample of 33 patients with wound, burn or ulcers infected with P. aeruginosa all treated with topical acetic acid., $5,12,13$ The concentration of acetic acid ranged from $0.5-5 \%$ and duration ranged from 7-28 days. P. aeruginosa was eradicated from the infection sitein $84.8 \%$ of the cases $(28 / 33)$.

\section{Discussion}

Based on the literature review, it is evident that there is a lack of randomized controlled trials evaluating the utility of topical antimicrobials or antiseptics. As such, the question remains what is the best approach to treatment for pseudomonas infections.

In addition to the studies included in this review, there were several case reports that described the use of topical antiseptics. One report done by Nagoba et al. described the use of acetic acid to successfully treat P. aeruginosa wound infection as the sole antiseptic. ${ }^{14}$ There were three case reports demonstrating topical silver dressings as effective treatment for $P$. aeruginosa wound infection. These cases either reported the successful eradication of infection or it was implied by full recovery in absence of repeat wound cultures. ${ }^{16,17}$ Two cases used silver sulfadiazine and one used Actisorb Plus 25 (Johnson \& Johnson, New Jersey, USA) to effectively treat pseudomonas wound and burn infections. ${ }^{16,17}$ The research conducted by Stanford et al., prophylactically treated all patients presenting with burns with $1 \%$ silver sulfadiazine. ${ }^{16}$ Three patients included in the study had established pseudomonas infection prior to treatment that subsequently converted to negative cultures. Additionally, Stanford et al., reported a case of a 40-year-old female with burns (65\% total body area) treated with $1 \%$ silver sulfadiazine that was discontinued in preparation for skin grafts, after which she developed pseudomonas infection. ${ }^{16}$ She was then effectively treated with $1 \%$ silver sulfadiazine. One case series by Tessler et al. that described a 75\% mortality rate in four patients despite antibiotic and silver nitrate therapy secondary to burn infections.
In these cases the patients sustained significant burns (total body area ranged from 39-88\%) complicated by pseudomonas infection. The details of the topical silver nitrate treatment were not clear, and therefore did not meet inclusion criteria in this review. However they found that $0.5 \%$ topical silver nitrate might keep wounds deceptively clean and non-malodorous despite infection.

A Cochrane review evaluating the utility of silver treatments and dressings for treatment of contaminated or infected wounds only found 3 randomized control trials. ${ }^{18}$ These randomized trials were not specific to $P$. aeruginosa infection and were limited by short follow-up periods and methodological issues regarding how wound healing was measured. This study reported that silver-containing dressings did not significantly increase wound healing as compared to standard dressings or best local practices. Similarly there were no significant differences in pain, patient satisfaction, and length of hospital stay or cost. The authors concluded that there is insufficient evidence to recommend silver-containing dressings for treatment of infected or contaminated chronic wounds. ${ }^{18}$ Another paper recommended the use of mafenide acetate for the treatment of superficial $P$. aeruginosa burn infection, with debridement and subsequent application of 5\% mafenide acetate for invasive burn wound infections caused by $P$. aeruginosa. ${ }^{10}$ However, there was no data to support these recommendations from clinical trials or from the centre where these practices were employed.

There are several studies that evaluate topical antimicrobials for prophylaxis of infection to reduce the bioburden of open wounds and burns. ${ }^{1,9,19,20}$ Prophylactic silver based topical treatments are most commonly studied and used for limiting bacterial proliferation. There does not appear to be any superiority between different types of silver topical antimicrobials. ${ }^{9,19,20}$

However, an in vivo study supported the use of silver sulfadiazine over silver nitrate solution $(0.5 \%)$ or mafenide (10\%) as prophylaxis against infection in burned mice. ${ }^{21}$ Assessing other antimicrobials, one 


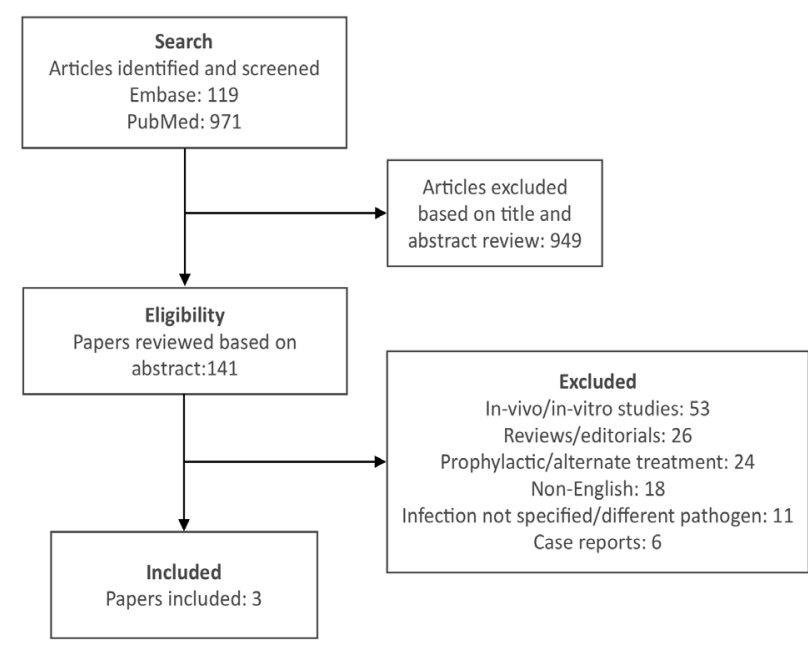

Figure 1. Search strategy used to identify appropriate papers demonstrating topical antiseptic therapy for Pseudomonas aeruginosa infections.

study done by MacMillan et al. demonstrated that mafenide and silver based topical treatment might reduce septic complications and mortality after sustaining thermal injuries. ${ }^{19}$ Comparing these topical treatment modalities, the authors concluded that these agents can be used interchangeably for prophylaxis and that one treatment did not demonstrate superiority.

Topical treatments have an important theoretical utility, while infected dead tissue from burns or other types of wounds do not benefit from the systemic antibiotic treatments with limited vascular supply. These dressings act as an adjunct to surgical debridement, help to absorb exudate and have antimicrobial action. ${ }^{15}$ Reviews and editorials report that topical treatment for active wound infection controls $P$. aeruginosa colony forming units and hastens the infectious course and time to recovery. However, this critical review demonstrates the limited clinical evidence to support this treatment in practice. The use of these topical agents is important to help decrease the use of systemic antibiotics and decrease subsequent adverse effects of these antibiotics. It is important to prevent the promotion of resistant microorganisms by limiting systemic antibiotic use with the use of broadspectrum topical agents. Acetic acid added topically to burn wounds acts locally against multi-drug resistant strains of $P$. aeruginosa and thus, could help to avoid these complications by reducing the need for systemic antibiotics. This would need to be tested definitively through larger, more rigorous trials.

The few clinical trials conducted demonstrated that acetic acid offers a nontoxic, well tolerated, inexpensive and efficient approach to wound infection complicated with $P$. aeruginosa. ${ }^{5,12,13}$ Similarly, in vitro studies have shown that $0.5 \%$ acetic acid was bactericidal to all isolated bacteria from cultured pseudomonas wound infections, with multiple drug resistance patterns. ${ }^{11}$ This is important with the increasing antibiotic resistance of bacterial skin flora, especially in critical care patients such as those with burn wounds, and the need for antibiotic stewardship. Also, it is difficult for systemic antibiotics to appropriately treat $P$. aeruginosa infections due to its ability to form resistant biofilms, which topical agents may be more effective. ${ }^{22}$ Madhusudhan prospectively randomized the use of acetic acid versus saline to treat chronic wounds infected with $P$. aeruginosa and found that those treated with acetic acid were cleared of pseudomonal infection earlier (4.5 versus 11.5 days). The acetic acid group had a higher proportion of multi-drug resistant $P$. aeruginosa, but the duration of treatment until infection was cleared was the same regardless of susceptibilities, as opposed to the saline treatment group, which took longer. ${ }^{22}$

To produce a $0.5 \%$ solution of acetic acid, one simply needs to dilute $1 \mathrm{~mL}$ of acetic acid into $199 \mathrm{~mL}$ of sterile normal saline. Sterile gauze is then soaked in this solution and applied to the burn wounds. Dressings are typically changed once or twice a day, depending on the amount of drainage of the wound itself. The limitations of acetic acid are that application of the dressings can be painful in higher concentrations, and it has been show in vitro to temporarily disrupt epithelialization, although this has not been replicated in human or animal experiments. ${ }^{22}$

\section{Conclusion}

The advantages of topical antiseptics are to prevent and treat resistant strains of pseudomonas. There are no clinical trials that directly compare the different topical antimicrobials. However, there is some limited evidence to support topical acetic acid treatment for active pseudomonas wound infection and some studies to support silver based therapies for the prophylaxis of infection. It is obvious from this review that further clinical trials are recommended to evaluate various topical antimicrobials to help guide treatment for $P$. aeruginosa infected wounds.

\section{References}

1. Leaper DJ. Silver dressings: their role in wound management. Int Wound J 2006;3(4):282-94.

2. MacMillan BG, Hill EO, Altemeier WA. Use of topical silver nitrate, mafenide, and gentamicin in the burn patient: a comparative study. Arch Surg 1967;95(3):472-81.

3. Nagoba BS, Selkar SP, Wadher BJ, Gandhi RC. Acetic acid treatment 
of pseudomonal wound infections: a review. J Infect Public Health 2013;6(6):410-5.

4. Steinstraesser L, Schubert C, Jacobsen F, Al-Benna S. Editorial: glycyrrhizin against multi-resistant bacteria? J Leukoc Biol 2010;87(1):7-8.

5. Sloss JM, Cumberland N, Milner SM. Acetic acid used for the elimination of Pseudomonas aeruginosa from burn and soft tissue wounds. J R Army Med Corps 1993;139(2):49-51.

6. Pruitt BA, McManus AT, Kim SH, Goodwin CW. Burn wound infections: current status. World J Surg. 1998;22(2):135-45.

7. Tessler R, Polk HC. Pseudomonas burn sepsis in patients treated with aqueous silver nitrate (0.5 percent). Surgery 1967;61(5):705-10.

8. Ollstein RN, McDonald C. Topical and systemic antimicrobial agents in burns. Ann Plast Surg 1980;5(5):386-92.

9. McDougall IA. Burns and the use of silver sulphadiazine. Aust N Z J Surg 1972;42(2):174-8.

10. Pruitt BA, Goodwin CW. Current treatment of the extensively burned patient. Surgery Annual 1983;15:331-64.

11. Mama M, Abdissa A, Sewunet T. Antimicrobial susceptibility pattern of bacterial isolates from wound infection and their sensitivity to alternative topical agents at Jimma University Specialized Hospital, South-West Ethiopia. Ann Clin Microbiol Antimicrob 2014;13:1.

12. Nagoba B, Wadher B, Kulkarni P, Kolhe S. Acetic acid treatment of Pseudomonal wound infections. Eur J Gen Med 2008;5(2):104-6.

13. Phillips I, Lobo AZ, Fernandes R, Gundara NS. Acetic acid in the treatment of superficial wounds infected by Pseudomonas aeruginosa. Lancet 1968;1(7532):11-4.
14. Nagoba BS, Deshmukh SR, Wadher BJ, Patil SB. Acetic acid treatment of pseudomonal postoperative wound infection. J Hosp Infect 1997;36(3):243-4.

15. Karonidis A, Delikonstantinou I, Tsoutsos D. Use of Actisorb* dressings over a skin-grafted infected wound. Burns 2011;37(2):360-1.

16. Stanford W, Rappole BW, Fox CL. Clinical experience with silver sulfadiazine, a new topical agent for control of pseudomonas infections in burns. J Trauma 1969:9(5):377-88.

17. Withers JN. Control of pseudomonas infections in burns with silver sulfadiazine. Hawaii Med J 1970;29(4):298-300.

18. Vermeulen H, Van Hattem JM, Storm-Versloot MN, Ubbink DT. Topical silver for treating infected wounds. Cochrane Database Syst Rev 2009;1:CD005486.

19. MacMillan BG, Hill EO, Altemeier WA. Use of topical silver nitrate, mafenide, and gentamicin in the burn patient: a comparative study. Arch Surg 1967;95(3):472-81.

20. Ollstein RN, Symonds FC, Crikelair GF, Pelle L. Alternate case study of topical sulfamylon and silver sulfadiazine in burns. Plast Reconstr Surg 1971;48(4):311-7.

21. Fox CL. Silver sulfadiazine--a new topical therapy for Pseudomonas in burns: therapy of Pseudomonas infection in burns. Arch Surg 1968;96(2):184-8.

22. Madhusudhan VL. Efficacy of $1 \%$ acetic acid in the treatment of chronic wounds infected with Pseudomonas aeruginosa: prospective randomised controlled clinical trial. Int Wound J 2015. doi: 10.1111/iwj.12428.

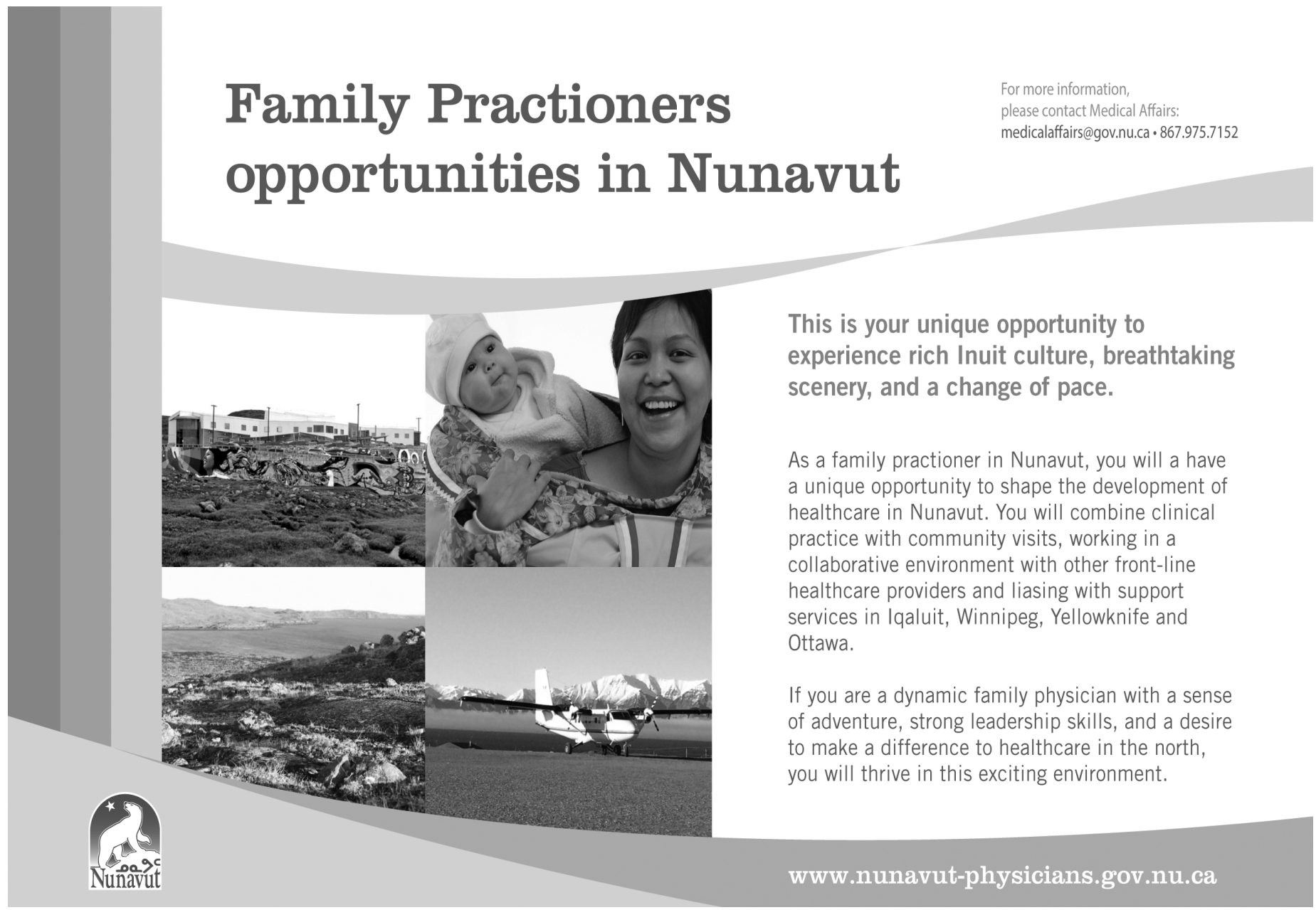

